\title{
Classification of the Excitation Location of Snore Sounds in the Upper Airway by Acoustic Multifeature Analysis
}

\author{
Kun Qian, Student Member, IEEE, Christoph Janott, Student Member, IEEE, \\ Vedhas Pandit, Student Member, IEEE, Zixing Zhang, Member, IEEE, Clemens Heiser, \\ Winfried Hohenhorst, Michael Herzog, Werner Hemmert, Senior Member, IEEE, \\ and Björn Schuller, Senior Member, IEEE
}

\begin{abstract}
Objective: Obstructive sleep apnea (OSA) is a serious chronic disease and a risk factor for cardiovascular diseases. Snoring is a typical symptom of OSA patients. Knowledge of the origin of obstruction and vibration within the upper airways is essential for a targeted surgical approach. Aim of this paper is to systematically compare different acoustic features, and classifiers for their performance in the classification of the excitation location of snore sounds. Methods: Snore sounds from 40 male patients have been recorded during drug-induced sleep endoscopy, and categorized by Ear, Nose \& Throat (ENT) experts. Crest Factor, fundamental frequency, spectral frequency features, subband energy ratio, mel-scale frequency cepstral coefficients, empirical mode decomposition-based features, and wavelet energy features have been extracted and fed into several classifiers. Using the ReliefF algorithm, features have been ranked and the selected feature subsets have been tested with the same classifiers. Results: A fusion of all features after a ReliefF feature selection step in combination with a random forests classifier showed the best classification results of $78 \%$ unweighted average recall by subject independent validation. Conclusion: Multifeature analysis is a promising means to help identify the anatomical mechanisms of snore sound generation in individual subjects. Significance: This paper describes a novel ap-
\end{abstract}

This work was supported in part by the China Scholarship Council, in part by the European Union's Seventh Framework, and in part by the Horizon 2020 under Grant 338164 (ERC Starting Grant iHEARu), and under Grant 645378 (ARIA-VALUSPA). Asterisk indicates corresponding author.

${ }^{*} \mathrm{~K}$. Qian is with the Machine Intelligence and Signal Processing Group, MMK, Technische Universität München, Munich 80333, Germany (email: andykun.qian@tum.de).

C. Janott and W. Hemmert are with the Institute for Medical Engineering, Technische Universität München.

V. Pandit and Z. Zhang are with the Chair of Complex and Intelligent Systems, University of Passau.

C. Heiser is with the Department of Otorhinolaryngology/Head and Neck Surgery, Technische Universität München.

W. Hohenhorst is with the Clinic for ENT Medicine, Head and Neck Surgery, Alfried Krupp Krankenhaus.

M. Herzog is with the Clinic for ENT Medicine, Head and Neck Surgery, Carl-Thiem-Klinikum Cottbus.

B. Schuller is with the Department of Computing, Imperial College London, and the Chair of Complex and Intelligent Systems, University of Passau. proach for the machine-based multifeature classification of the excitation location of snore sounds in the upper airway.

Index Terms-Drug-induced sleep endoscopy (DISE), multifeature analysis, obstructive sleep apnea (OSA), snore sound classification.

\section{INTRODUCTION}

W ITH a prevalence of $13 \%$ (men) and 6\% (women) in the U.S. population [1], obstructive sleep apnea (OSA) is a chronic disease that can severely affect health and quality of life. OSA is defined as a syndrome with cessation or reduction of airflow during sleep due to complete (apnoea) or partial (hypopnea) collapse of the upper airway for more than $10 \mathrm{~s}$ and with five or more episodes per hour in sleep [2]. It is usually associated with a decrease in oxyhemoglobin saturation [2]. When untreated, OSA can be among other symptoms, result in daytime sleepiness and morning headache [3]. Furthermore, it is an independent risk factor for cardiovascular diseases, stroke, hypertension, myocardial infarction, and is associated with diabetes and vulnerability to accidents [4], [5].

Loud snoring, as a typical symptom of OSA, is reported in more than $80 \%$ of OSA patients [6]. The acoustic properties of snoring have been analyzed by the researchers in acoustics and otorhinolaryngology with the aim of developing methods to replace or complement the gold standard for the diagnosis of OSA polysomnography (PSG) [7]. Works by pioneers have shown that methods based on snoring sound analysis can reach the sensitivities and specificities up to $90 \%$ and accuracies up to $80 \%$ in the detection of OSA [8]. Even though based on small populations (generally between 5 and 60 subjects) [8], the results are promising and encouraging.

Due to the multifactorial mechanisms of snore sound $(\mathrm{SnS})$ generation, and depending on the individual anatomy, surgical options for OSA differ and include, among others, tonsillectomy or tonsillotomy, uvulotomy, uvulopalatopharyngoplasty, soft palate stiffening, tongue base suspension, hypoglossal nerve stimulation, mandibular advancement, epiglottectomy, and hyoid suspension [9]. Especially in severe OSA, a combination of several surgical treatments at different anatomic levels (multilevel surgery) [10] is often used. The analysis of the individual 
anatomical site of snoring sound generation, and of the obstruction mechanism can lead to a targeted, and less invasive surgical approach.

Drug-induced sleep endoscopy (DISE) is increasingly used to identify the location and form of vibrations and obstructions [11]. However, DISE is time consuming, costly, and straining for patients. Further, it cannot be performed in natural sleep. Another method to identify the location of obstruction in the upper airway is multichannel pressure measurement [12]-[14]. Here, a thin tube with multiple pressure sensors is introduced into the upper airway. The pattern of pressure changes during the breathing of the different sensors allows a determination of the obstruction location during an apnoeic or hypopnoeic event. An advantage of this method is that it can be used in natural sleep. However, the tube within the upper airway is not tolerated by every patient. Acoustic analysis could be an alternative to determine the vibration mechanisms within the upper airway, which is easier for doctors and patients.

Fewer studies exist on how to determine the location and form of vibration and obstruction in the upper airway from the acoustic properties of SnSs. Miyazaki et al. [15] adopted fundamental frequency to distinguish $\mathrm{SnS}$ generated by soft palate, tonsils/tongue base, combined type (both palate, and tonsils/tongue base), and the larynx. Based on the examination of 75 adult patients, they concluded that the average value of fundamental frequency was $102.8,331.7,115.7 \mathrm{~Hz}$, and around $250 \mathrm{~Hz}$ in the corresponding sites mentioned above, respectively. Hill et al. found the crest factor, the ratio of peak to root-mean-square (rms) value of a time-varying signal, to be significantly higher for palatal snorers $(p<0.01$, Student- $t$ or Mann-Whitney tests) in 11 patients [16]. Agrawal et al. found that the SnS generated by palate and tongue were respectively characterized by low and high peak frequency (137 and $1243 \mathrm{~Hz}$ ), while epiglottic snores occurred at $490 \mathrm{~Hz}$ and tonsillar snores at $170 \mathrm{~Hz}$ within a population of 16 subjects [17]. Beeton et al. proposed a combination of a two-means clustering method and the statistical dimensionless moment coefficients of skewness and kurtosis to discriminate palatal and nonpalatal SnS collected from 15 patients [18]. They indicated that the statistical moment coefficients demonstrate a method of measurement of the peakedness and symmetry of the impulse.

The studies mentioned above are focused on evaluating certain well-selected acoustic features for their sensitivity to the anatomical mechanisms of snoring sound generation or upper airway obstruction. The comparisons and results are based on statistical analysis, and basic signal observation. The application of multifeature analysis methods to this problem has been proposed [19]; however, advanced signal processing methods, and machine learning models have not yet been used for this purpose. In our approach, we combine the methods used previously, and introduce new features within advanced classification techniques. The structure of this paper is as follows: In Section II, we present the data acquisition system and the methods for multifeature analysis-based classification. Detailed experimental results are given in Section III. Finally, we discuss the findings in Section IV, and provide a conclusion in Section V.
TABLE I

DEMOGRAPHIC INFORMATION OF SUBJECTS. BMI: BODY MASS INDEX. AHI: APNEA HYPOPNEA INDEX

\begin{tabular}{lccc}
\hline \hline & Mean & Std. Dev. & Range \\
\hline Age (years) & 47.4 & 11.5 & $26-71$ \\
BMI (kg/m $\left.{ }^{2}\right)$ & 26.9 & 3.1 & $21.2-38.4$ \\
AHI (events/h) & 21.7 & 12.8 & $1.3-59.1$ \\
\hline \hline
\end{tabular}

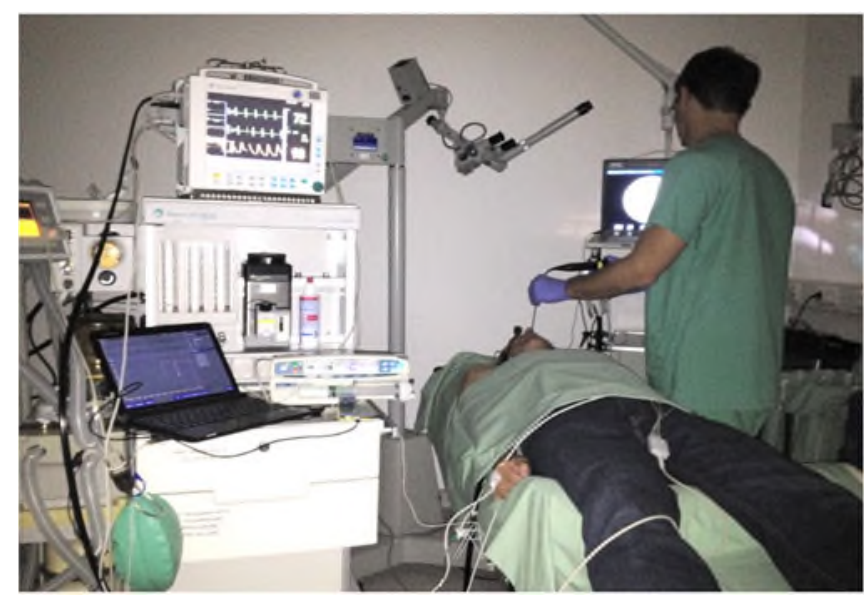

Fig. 1. Example of the DISE clinical setting in Munich. The video of the upper airway was recorded using a flexible nasopharyngoscope (Storz, Germany at the Munich and Halle sites and Olympus, Germany, at the Essen site) connected to a video recording system (Telepack X, Storz, Germany, at the Munich site; AIDA, Storz, Germany, at the Halle site; rpSzene, Rehder/Partner, Hamburg, Germany, at the Essen site). The audio signal was simultaneously recorded using a microphone connected to the same recording system. Audio and video information was stored in the same file.

\section{Materials AND MEthods}

\section{A. SnS Data Acquisition and Labeling}

This study is approved by the ethic committee of Klinikum rechts der Isar, Technische Universität München, Germany. We used $\mathrm{SnS}$ data from 40 male subjects, which were diagnosed with primary snoring or OSA through a PSG. The demographic data for the subjects are shown in Table I.

In addition to PSG, DISE was performed in all subjects in order to determine adequate surgical intervention measures. DISE videos were recorded at Klinikum rechts der Isar, Munich, Germany, at Alfried Krupp Hospital, Essen, Germany, and at University Hospital Halle (Saale), Germany, using a flexible nasopharyngoscope (see Fig. 1 as an example of the clinical setting). Audio information was recorded in parallel using a headset microphone (in Munich), or a handheld microphone (in Essen and Halle), respectively, and synchronously stored in the same file. Based on the video and audio recordings, the locations of sound generation were categorized by an ENT expert based on the VOTE classification [20]. VOTE is a popular classification, which distinguishes four levels within the upper airway: the level of the velum (V), the oropharyngeal area including the palatine tonsils $(\mathrm{O})$, the tongue base $(\mathrm{T})$, and the epiglottis (E) (see Fig. 2). Only recordings that showed a clearly identifi- 


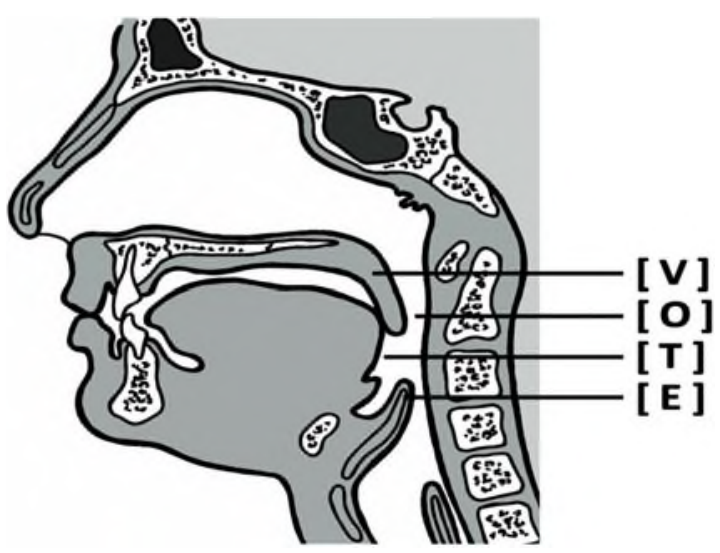

Fig. 2. Corresponding positions of the VOTE classification in the upper airway. "V" represents the level of the velum. "O" represents the oropharyngeal area. "T" represents the tongue base. "E" represents the level of the epiglottis.

able single source of snoring sound have been included. Snoring events with mixed forms (several vibration locations) or unclear source of vibration were excluded. From each included recording, three to five snoring events, which showed no obstructive disposition, have been manually selected. These snoring events have then been extracted from the audio data stream, and labeled based on the VOTE classification. In fact, "snore site" and "obstruction site" in the upper airway are two different definitions, which may or may not coincide in individual patients. In this study, we exclusively focus on the determination of the site of vibration as a cause for the generation of SnSs.

Out of the 40 subjects, $11,11,8$, and 10 subjects were categorized to be $\mathrm{V}, \mathrm{O}, \mathrm{T}$, and E-type snorers, respectively. Between one and five snoring events per type were extracted per subject. In total, we used 164 snoring events (41 episodes for each type of $\mathrm{SnS}$, length ranging from 0.728 to $2.495 \mathrm{~s}$ with an average of $1.498 \mathrm{~s}$ ). We segmented the events into single segments for further feature extraction and machine learning. Every segment has a length of $200 \mathrm{~ms}$ and neighboring segments have an overlap of $50 \%$. We performed a subject-independent validation to evaluate the performance of our trained classifiers. As indicated by Roebuck et al. [8], previous works on snoring audio analysis have not been based on independent test datasets. In order to achieve substantiated results with a practical relevance, we use subject-independent testing sets in our study. We randomly separated the 40 subjects' data into the train, development (dev), and test sets within the proportion of $60 \%, 20 \%$, and $20 \%$ of the total dataset. The number of segments and independent subjects for each set are shown in Table II. The segments are divided into frames of $64 \mathrm{~ms}$ length and an overlap of 50\%. Features and statistical functionals are applied to each frame in every segment and all attribute information is stored for further machine learning steps.

\section{B. Feature Extraction}

Until most recent, the lion's share of the work done in multifeature snoring analysis is focused on finding and evaluating suitable acoustic feature sets [7] to identify obstructive events,
TABLE II

NUMBER OF SEGMENTS (INDEPENDENT SUBJECTS) FOR EACH SNORE-TYPE AND DATASET

\begin{tabular}{lcccc}
\hline \hline & Train & Dev & Test & $\sum$ \\
\hline V-Type & $363(7)$ & $104(2)$ & $152(2)$ & $619(11)$ \\
O-Type & $326(7)$ & $125(2)$ & $122(2)$ & $573(11)$ \\
T-Type & $289(4)$ & $90(2)$ & $78(2)$ & $457(8)$ \\
E-Type & $323(6)$ & $96(2)$ & $148(2)$ & $567(10)$ \\
$\sum$ & $1301(24)$ & $415(8)$ & $500(8)$ & $2216(40)$ \\
\hline \hline
\end{tabular}

to distinguish between primary snoring and OSA, or to estimate the severity of OSA. Abeyratne et al. proposed a multifeature analysis method built on combined models of pitch, total airway response estimators, and Mel-frequency cepstral coefficients (MFCCs, 0-12), and achieved 89.3\% sensitivity with $92.3 \%$ specificity in OSA detection [21].

Fig. 3 illustrates the waveforms and the corresponding spectrograms of typical V, O, T, and E-type SnS episodes. We can see that the main energy components in three of the classes are concentrated in the frequency area below around $5000 \mathrm{~Hz}$. Energy and spectral distribution characteristics are similar, except for the Type T, which shows higher energy content above $2500 \mathrm{~Hz}$ compared to the other three.

Motivated by the results of the multifeature analysis methodology [21], combined with the basic spectral analysis of the four types of $\mathrm{SnS}$, we propose nine basic acoustic feature sets, and systematically explore, and compare their performance in the classification of SnSs based on the VOTE system.

1) Crest Factor: Hill et al. in 1999 proposed the use of the crest factor [16]; we redefine it as shown in our previous study [22]

$$
\text { Crest Factor }=\frac{V_{90}}{V_{\text {rms }}}
$$

where $V_{90}$ is the 90th centile maximum absolute value in the digitized sound epoch, and $V_{\text {rms }}$ is the root-mean-square of the amplitude values (between the 10th and 90th centile maximum absolute values) in one epoch. Elimination of the lowest (below 10th) and highest (above 90th) values is done to minimize the impacts of both random and quantization noise.

2) Fundamental Frequency (FO): We estimate the F0 of SnS with an algorithm based on spectrum shifting on a logarithmic frequency scale and calculating the subharmonic-toharmonic ratio, which was proposed by Sun [23].

3) Formants: In our study, an 18th-order linear predictive coding (LPC) is performed to estimate the formants. Like in speech analysis, the LPC parameters are determined via the Yule-Walker autoregressive method along with the LevinsonDurbin recursive procedure [24]. Then, the formant frequencies can be estimated from the angles of the positive values of the complex roots in an all-pole model as follows:

$$
H_{(z)}=\frac{1}{1-\sum_{q=1}^{p} \alpha_{q} z^{-q}}
$$

where $\alpha_{q}(q=1,2,3, \ldots, p)$ are the LPC parameters. Subsequently, we extract the first three formant frequencies (i.e., F1, 

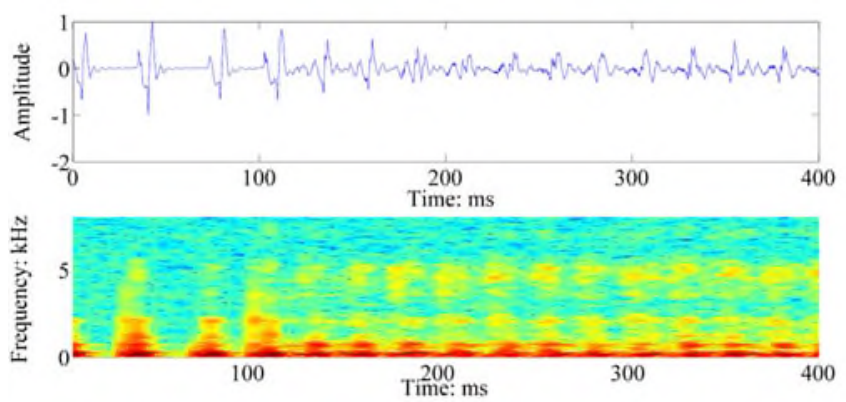

(a)
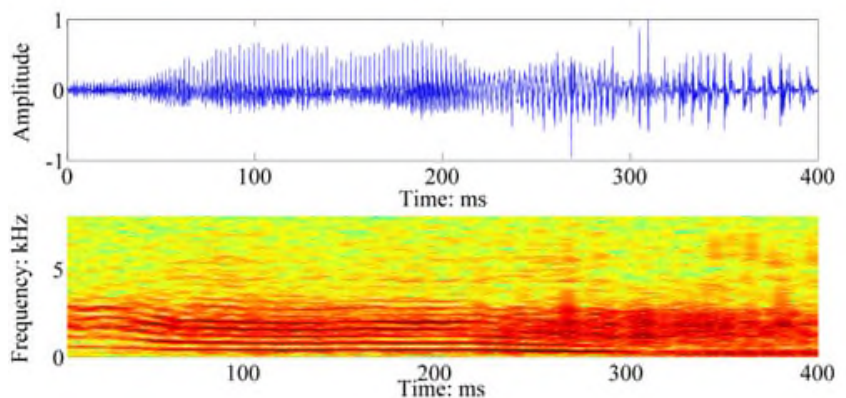

(c)
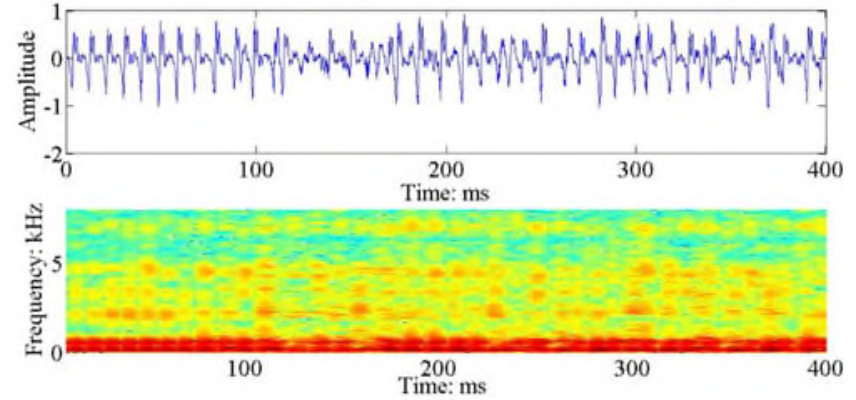

(b)
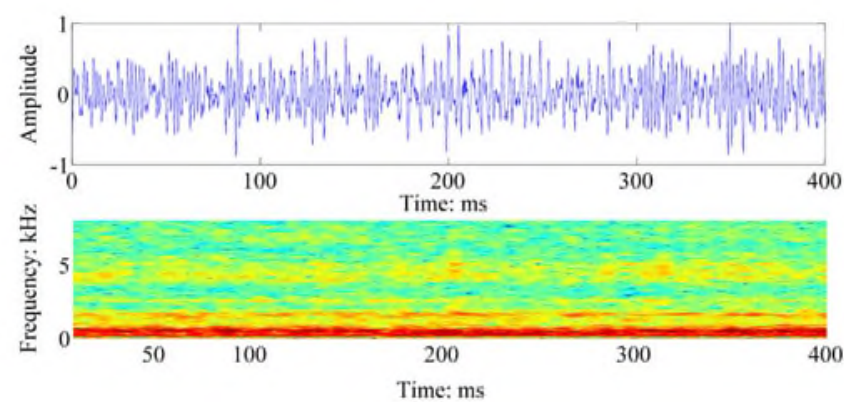

(d)

Fig. 3. Waveforms and spectrograms of typical VOTE SnS events. (a) V (velum) typical snoring event. (b) O (oropharyngeal) typical snoring even. (c) $\mathrm{T}$ (tongue base) typical snoring event. (d) $\mathrm{E}$ (epiglottis) typical snoring event.

F2, and F3), and the corresponding amplitude energies to create a formants feature set.

4) Spectral Frequency Features: The spectrum of $\mathrm{SnS}$ carries vital information on the state of the upper airway [25]. Certain frequency features such as peak frequency, center frequency, and mean frequency, were studied both on the diagnosis of OSA [21] and distinction of the snore site [17]. In our previous work [26], we found that spectral frequency features (SFF) can achieve a good performance on the classification of inspiration related $\mathrm{SnS}$. Here, we define $F_{\text {center }}$, and $F_{\text {peak }}$, respectively, as the half and maximum point in the full spectrum of the SnS in [17], $F_{\text {mean }}$ is defined as follows:

$$
F_{\text {mean }}=\frac{\sum_{f_{i}=0}^{f_{c}} f_{i} S\left(f_{i}\right)}{\sum_{f_{i}=0}^{f_{c}} S\left(f_{i}\right)}
$$

where $f_{c}$ is the cutoff frequency of the SnS spectrum (in our study $f_{c}$ is $\left.8 \mathrm{kHz}\right) . S\left(f_{i}\right)$ is the absolute amplitude of the spectrum at the frequency of $f_{i} \mathrm{~Hz}$. The spectrum calculations in this study are based on the fast Fourier transform (FFT). In addition, we define the $F_{\text {mean }}$ of the $1000 \mathrm{~Hz}$ subband spectrums as

$$
F_{\text {mean }(j)}=\frac{\sum_{f_{i}=1000(j-1)}^{1000 j} f_{i} S\left(f_{i}\right)}{\sum_{f_{i}=1000(j-1)}^{1000 j} S\left(f_{i}\right)}
$$

where $j=1,2,3, \ldots, 8$. Thus, we obtain detailed information on the spectral energy distribution in the subbands of $\mathrm{SnS}$.

5) Power Ratio: Power ratio (PR) compares the relative amount of power emanating below and above a set frequency [17]. Some researchers chose the frequency at
$800 \mathrm{~Hz}$ [27], and others chose it to be $750 \mathrm{~Hz}$ [17]. In this study, we use the PR at the frequency of $800 \mathrm{~Hz}$ and define it as

$$
\mathrm{PR}_{800}=\lg \frac{\sum_{f_{i}=0}^{800}\left(S\left(f_{i}\right)\right)^{2}}{\sum_{f_{i}=800}^{f_{c}}\left(S\left(f_{i}\right)\right)^{2}}
$$

6) Subband Energy Ratio: The subband energy ratio (SER) describes the relative energy distribution in subbands of the $\mathrm{SnS}$ spectrum. It had been demonstrated to be efficient in snore/nonsnore classification [28], [29]. We extract a 1000-Hz SER feature set as

$$
\operatorname{SER}_{1000}(j)=\frac{\sum_{f_{i}=1000 j-1}^{1000 j}\left(S\left(f_{i}\right)\right)^{2}}{\sum_{f_{i}=0}^{f_{c}}\left(S\left(f_{i}\right)\right)^{2}}
$$

where $j=1,2,3, \ldots, 8$.

7) Mel-Scale Frequency Cepstral Coefficients: MFCCs have long been demonstrated to be efficient features for speech recognition [30]. In our previous study [31], we found that MFCCs can outperform other spectral features on classification of $\mathrm{SnS}$. In this study, we extract $13 \mathrm{Mel}$ cepstral coefficients (MFCCs 0-12) obtained from SnS passing 27 triangular Mel filter banks.

8) Empirical Mode Decomposition-Based Features: SnS are a typical kind of nonstationary signal [7]. Therefore, FFT-based methods are not suitable to reveal detailed information on the variation of the $\mathrm{SnS}$ in the time domain. Empirical mode decomposition (EMD), based on choosing basis functions, is adaptive to characterize nonstationary signals [32]. Motivated by the performance of EMD-based features (EMDF) for classification of roller bearing fault vibration signals [33], we extract the subband EMD energy ratio $\operatorname{EMD}_{\text {ratio }}(k)=E_{k} / E$. $E_{k}$ is the energy (sum of squares) of the $k$ th level intrinsic mode 
functions decomposed by EMD from the $\mathrm{SnS}$. $E$ is the total energy of the whole $\mathrm{SnS}$ within EMD (the residual is eliminated). In addition, we calculate the entropy of the $\mathrm{EMD}_{\text {ratio }}$ as

$$
H_{\mathrm{EMD}_{\text {ratio }}}=-\sum_{k=1} \operatorname{EMD}_{\text {ratio }}(k) \lg \left(\operatorname{EMD}_{\text {ratio }}(k)\right) \text {. }
$$

9) Wavelet Energy Features: It is known that wavelet transform (WT) is a useful tool to analyze nonstationary signals [34]. In 2007, Matsiki et al. studied how to use WT-based methods to analyze $\mathrm{SnS}$ of OSA patients [35]. Ng et al. used WT to enhance the snore signal from a noisy environment for improving the feature extraction [36]. Khushaba et al. [37] presented a wavelet-packet-based feature extraction algorithm and adopted it to classify five different drowsiness levels based on Electroencephalography, Electrooculography, and Electrocardiography signals. In this study, we introduce this wavelet packet transform (WPT) method into $\mathrm{SnS}$ multifeature extraction.

The core technique of the algorithm proposed by Khushaba et al. is the WPT, introduced by Coifman et al. [38]. The WPT could be understood as a tree of subspaces, where $\Phi_{0,0}$ is the root node. The signal space $\Phi_{l, m}$ ( $l$ is the level of the decomposition process and $m$ is the subband index) is decomposed into two orthogonal subspaces level by level: $\Phi_{l+1,2 m}$ and $\Phi_{l+1,2 m+1}$, namely, the approximation space, and the detail space [39].

This decomposition process is done by dividing an orthogonal basis $\Omega_{l}\left(t-2^{l} m\right)_{l, m \in Z}$ from $\Phi_{l, m}$ into two new orthogonal bases: $\Omega_{l+1}\left(t-2^{l+1} m\right)_{l, m \in Z}$ from $\Phi_{l+1,2 m}$ and $\Psi_{l+1}\left(t-2^{l+1} m\right)_{l, m \in Z}$ from $\Phi_{l+1,2 m+1}$, respectively, where, $\Omega_{l, m}(t)$ and $\Psi_{l, m}(t)$ are wavelet functions [34] (we select "sym3" of the "Symlets" wavelet function family due to its best performance in our previous experiments [40]). A construction of normalized filter bank energy is defined as

$$
E_{\Phi_{l, m}}=\sqrt{\frac{\sum_{n}\left(\mathbf{w}_{l, m, n}\right)^{2}}{N_{m}}}, m=0,1,2, \ldots, 2^{l-1}
$$

where $\mathbf{w}_{l, m}$ represent the WPT coefficients calculated from the signal at the subspace $V_{l, m}$, and $N_{m}$ is the number of wavelet coefficients in the $m$ th subband. Therefore, $E_{\Phi_{l, m}}$ denotes the normalized bank filter energy in $m$ th subband at the $l$ th decomposition level. Due to the origination of these features from WPT-based coefficients, we call them WPT Energy (WPTE) features.

As a complementary feature set, we use the WT to define a percentage-like WT-based Energy (WTE) feature as

$$
\hat{E}_{\hat{\mathbf{\Phi}}_{l}}=\frac{\left(\hat{\mathbf{w}}_{l}\right)^{2}}{\sum_{l=1}^{L_{\max }}\left(\hat{\mathbf{w}}_{l)^{2}}\right.} \times 100
$$

where $\hat{\mathbf{w}}_{l}$ are the coefficients generated by WT at the $l$ th decomposition level. In addition, the mean variance, waveform length (the sum of absolute differences), and the entropy are calculated from the base by (9).

WT decomposes only the approximation part, using low-pass filters, whereas WPT decomposes both the approximation and

\footnotetext{
${ }^{1}$ The wavelet function names in this paper are all identical to the ones given in the Wavelet Toolbox of MATLAB by MathWorks(http://www.mathworks.com/products/wavelet/).
}

TABLE III

Frame-Based Feature Sets and Statistical Functionals

\begin{tabular}{ll}
\hline \hline Frame-based feature sets (338) & Statistical functionals (9) \\
\hline Crest Factor (1), FO (1), & max, min, mean, \\
Formants (6), SFF (11), & range, standard deviation, \\
PR 800 (1), SER (8), & slope, bias (linear \\
MFCCs (13), EMDF (10), & regression approximation) \\
WEF (287) & skewness, kurtosis \\
\hline \hline
\end{tabular}

the detail part (low pass and high pass filtering). All WPTE and WTE features are modified with a logarithmic operator. We generate $2^{L_{\max }+1}-1$ WPTE related features, and $4 \times\left(L_{\max }+\right.$ 1) WTE related features, where $L_{\max }$ is the maximum level for wavelet decomposition. We then fuse all WPTE and WTE features in one combined feature set. Since these are all energy related features, we call the resulting feature set "Wavelet energy features (WEF)." Here, $L_{\max }$ is set to be 7 , therefore, we extract $255+32=287$ descriptors in total.

10) Statistical Functionals: In order to evaluate the nonstationary characteristics of the material, the differences between the frames within a segment are taken into account [7]. Motivated by the success of our large scale feature extraction toolkit, openSMILE [41], we implement statistical functionals into our $\mathrm{SnS}$ feature extraction. After calculating the framebased features using the algorithms described above, the statistical functionals are applied to each frame in every segment. The whole attribute information of this segment is used for the machine learning process. Detailed information of this technique can be found in [41]. The basic frame-based feature sets and the statistical functionals are listed in Table III.

\section{Machine Learning Models}

$\mathrm{SnS}$ recognition based on the VOTE classification is a fourclass classification task. We select and compare seven machine learning models, which we have chosen based on their popularity, diversity, and abilities. $K$-nearest neighbors $(K-\mathrm{NN})$ and linear discriminant analysis (LDA) are chosen since their probabilistic models are built for and they are frequently applied in biomedical signal processing tasks [42]. Models with a mature theoretical foundation like feedforward neural networks (FNN) [43]), support vector machines (SVM) ${ }^{2}$ [44], and random forests (RF) [45] (an ensemble classifier [46]) are also used in our experiments. Further, extreme learning machines (ELM) [47] and the kernel-ELM (KELM) [48], one recent popular fast and accurate classifier models, are explored.

\section{ReliefF Feature Selection}

To achieve higher classification accuracy and to understand how the different features contribute to the machine learning models, we add a feature selection phase into our classifier optimization process. Motivated by the success of our previous study [26], [31], we employ the ReliefF algorithm for feature

\footnotetext{
${ }^{2}$ The SVM classifier is implemented by the frequently-used, and mature toolkit LIBSVM [49].
} 
ranking and selection. Unlike principle component analysis, ReliefF retains the original physical meaning of each feature set in the vector. That is, we can further use these information of the ranked features to find the relationship between feature properties and anatomical characters, which is significant in our study.

Proposed by Kira and Rendell [50], Relief is a feature selection algorithm used in binary classification, which is repeated $t$ times to update the weight vector (initially it is set to be zeros) as follows:

$$
W_{a}=W_{a}-\left(x_{a}-\text { nearHit }_{a}\right)^{2}+\left(x_{a}-\text { nearMiss }_{a}\right)^{2}
$$

where nearHit ${ }_{a}$ is the closest same-class segment within the $a$ th feature to a randomly selected segment $x$. Likewise, nearMiss ${ }_{a}$ is the closest different-class segment. After $t$ times, each element of $W_{a}$ will be divided by $t$. Thus, the weight of any given feature will decrease if the distance of that feature to nearby segments of the same-class is longer than to nearby segments of a different-class, and increase in the reverse case. $W_{a}$ can be regarded as a reward and punishment factor due to the classification performance of each feature: Features with a high $W_{a}$ show a good performance for the classification task at hand. ReliefF is an extension of Relief, which can handle multiple classes and performs better with noisy data. It searches for $b$ (in our study, $b$ is empirically set to be 5) nearest hits and misses and averages their contributions for updating $W_{a}$, weighted with the prior probability of each class [51]. Since $W_{a}$ evaluates the quality of each feature, we can rank the features according to their performance, and select the best ones to construct a new subset of the original features. We define the Rank Ratio as

$$
\text { Rank Ratio }=\frac{\sum_{a=1}^{M} W_{a}^{+}}{\sum W_{a}^{+}}
$$

where $W_{a}^{+}$represents the positive weights of the features sorted in a descending order and $M$ is the number of features included in the subset. The features with negative weights $\left(W_{a}^{-}\right)$are eliminated. By testing the classifier with feature subsets of different sizes (based on different Rank Ratios), a feature subset for optimal classification performance can be identified, while at the same time, the size of the required feature set can be reduced.

\section{EXPERIMENTS AND RESULTS}

\section{A. Experimental Setup}

All experiments are done within the software environment of MATLAB R2016a by MathWorks. The grids of main parameters for each classifier model mentioned in Section II-C are listed in Table IV. We chose these parameters by empirical experiments to optimize the performance of classification on the development data. A statistical significance $p$ value is calculated by a onesided z-test [52].

\section{B. Experimental Baselines}

Before feeding them to the classifier model, all the features extracted from our $\mathrm{SnS}$ are normalized as follows:

$$
\hat{f}_{c, r}=\frac{f_{c, r}-\operatorname{Min}\left(\mathbf{F}_{c}\right)}{\operatorname{Max}\left(\mathbf{F}_{c}\right)-\operatorname{Min}\left(\mathbf{F}_{c}\right)}
$$

\begin{tabular}{|c|c|}
\hline Models & Main Parameters Setting \\
\hline$K-\mathrm{NN}$ & $\begin{array}{l}K: 1,10,20,30,40,50,60,70,80,90,100 ; \\
\text { distance metrics: "euclidean," "cityblock," "chebychev," } \\
\text { "correlation," "cosine," "hamming," "jaccard," } \\
\text { "minkowski," "seuclidean," "spearman"; }\end{array}$ \\
\hline LDA & discriminant type: linear; gamma: 0:0.05:1.00; \\
\hline SVM & $\begin{array}{l}\text { "linear kernel", "polynomial", "radial basis function", "sigmoid"; } \\
c \text {-value: } 10^{-5}, 10^{-4}, \ldots, 10^{4}, 10^{5} \text {; }\end{array}$ \\
\hline $\mathrm{RF}$ & $\begin{array}{l}\text { number of trees: } 2^{1}, 2^{2}, \ldots, 2^{9}, 2^{10} \\
\text { fraction for the treebagger: } 0.1: 0.1: 1.00\end{array}$ \\
\hline FNN & one hidden layer; size: $2^{1}, 2^{2}, \ldots, 2^{9}, 2^{10}$ \\
\hline ELM & $\begin{array}{l}\text { activation functions: "signmoidal," "sine," "hardlim," "tribas," } \\
\text { "radbas"; number of hidden neurons: } 2^{1}, 2^{2}, \ldots, 2^{14} ;\end{array}$ \\
\hline KELM & $\begin{array}{l}\text { kernels:"radial basis function," "linear," "polynomial," "wavelet"; } \\
\text { regularization coefficients: } 10^{-5}, 10^{-4}, \ldots, 10^{4}, 10^{5} \text {; }\end{array}$ \\
\hline
\end{tabular}

TABLE IV

Grids of MAIN PARAmETERS FOR EACH LEARNING MOdEL

where $f_{c, r}$ is the original $c$ th feature property for the $r$ th segment, $\mathbf{F}_{c}$ is the $c$ th feature vector, which includes the properties for all of the segments. Thus, the normalized feature property $\hat{f}_{c, r}$ will be limited into $[0,1]$.

In order to evaluate the performance of our method, we apply the unweighted average recall (UAR) defined as follows:

$$
\mathrm{UAR}=\frac{\sum_{\text {class=1 }}^{N_{\mathrm{MC}}} N_{\text {class, correct }} / N_{\text {class,all }}}{N_{\mathrm{MC}}} \times 100 \%
$$

where $N_{\text {class,correct }}$ and $N_{\text {class,all }}$ are the number of correctly recognized segments, and all segments in one certain class, respectively. $N_{\mathrm{MC}}$ is the total number of classes.

The UAR baselines of the different combinations of classifiers and feature sets are shown in Table V. We found that MFCCs within a $K$-NN classifier ( $K: 30$, distance metrics: "correlation”) achieve the best recognition rate of $76 \%(p<0.001)$. Of the nine feature sets, the novel wavelet-based WEF performs best with a mean UAR of $58 \%$ among all classifiers used. MFCCs score second best (mean UAR of $56 \%$ ), followed by SER (UAR of 55\%). The performance of $W E F$ and MFCCs is significantly better compared to the remaining six feature sets $(p<0.05)$. On the other hand, Crest Factor, F0, and $\mathrm{PR}_{800}$ did not show a good performance in our study.

\section{Feature Selection}

We use the ReliefF algorithm as described in Section II-D for the feature selection step. The different feature sets separately, as well as a complete combination of all features from each feature set $(A L L)$ are fed into the ReliefF process at Rank Ratio settings from 0.05 to 1.00 with increments of 0.05 to find the bestperforming combined subset. The best results of the features selected by ReliefF are shown in Table VI.

We find that, except for the Crest Factor that remains at $34 \%$ UAR, ReliefF can improve the mean performance of each feature set among different classifier models. In particular, for the combination of all feature sets, the mean performance significantly improves from $46 \%$ to $68 \%$ ( $p<0.001)$. For $S F F$ (45\% to $62 \%$ ), MFCCs (56\% to $68 \%$ ), and WEF (58\% to $66 \%$ ), the improvement after the feature selection step is also signif- 
TABLE V

UAR ([\%]) Achieved With Different Feature Sets and Classifiers Without Feature Selection

\begin{tabular}{lccccccccc}
\hline \hline & $K$-NN & LDA & SVM & RF & FNN & ELM & KELM & Mean & Std. dev. \\
\hline Crest Factor & 37 & 36 & 30 & 32 & 38 & 37 & 29 & 34 & \pm 3.7 \\
F0 & 32 & 37 & 34 & 27 & 36 & 31 & 32 & 33 & \pm 3.4 \\
Formants & 50 & 51 & 57 & 40 & 56 & 42 & 52 & 50 & \pm 6.5 \\
SFF & 60 & 59 & 25 & 66 & 55 & 25 & 25 & 45 & \pm 19.0 \\
PR800 & 35 & 39 & 36 & 35 & 39 & 36 & 41 & 37 & \pm 2.4 \\
SER & 62 & 56 & 51 & 66 & 50 & 44 & 53 & 55 & \pm 7.5 \\
MFCCs & 76 & 50 & 61 & 57 & 42 & 53 & 55 & 56 & \pm 10.5 \\
EMDF & 47 & 56 & 25 & 57 & 41 & 25 & 25 & 39 & \pm 14.5 \\
WEF & 59 & 53 & 53 & 59 & 56 & 59 & 64 & 58 & \pm 3.9 \\
ALL & 62 & 60 & 25 & 64 & 64 & 25 & 25 & 46 & \pm 20.1 \\
Mean & 52 & 50 & 40 & 50 & 48 & 38 & 40 & - & - \\
Std. dev. & \pm 14.3 & \pm 9.1 & \pm 14.3 & \pm 15.1 & \pm 9.7 & \pm 11.9 & \pm 14.8 & - & - \\
\hline \hline
\end{tabular}

TABLE VI

UAR ([\%]) Achieved With Different Feature Sets and Classifiers After the Feature Selection Step

\begin{tabular}{lccccccccc}
\hline \hline & $K$-NN & LDA & SVM & RF & FNN & ELM & KELM & Mean & Std. dev. \\
\hline Crest Factor & 35 & 36 & 31 & 36 & 39 & 30 & 30 & 34 & \pm 3.5 \\
F0 & 32 & 38 & 34 & 34 & 39 & 33 & 32 & 35 & \pm 2.8 \\
Formants & 54 & 50 & 55 & 50 & 57 & 50 & 57 & 53 & \pm 3.3 \\
SFF & 62 & 71 & 65 & 72 & 62 & 54 & 49 & 62 & \pm 8.4 \\
PR800 & 35 & 39 & 40 & 36 & 42 & 42 & 42 & 39 & \pm 2.9 \\
SER & 61 & 58 & 58 & 66 & 58 & 52 & 59 & 59 & \pm 4.2 \\
MFCCs & 76 & 64 & 62 & 70 & 73 & 66 & 64 & 68 & \pm 5.2 \\
EMDF & 52 & 55 & 60 & 58 & 56 & 47 & 54 & 55 & \pm 4.2 \\
WEF & 68 & 69 & 69 & 67 & 64 & 59 & 68 & 66 & \pm 3.6 \\
ALL & 74 & 73 & 74 & $\mathbf{7 8}$ & 71 & 45 & 58 & 68 & \pm 11.8 \\
Mean & 55 & 55 & 55 & 57 & 56 & 48 & 51 & - & - \\
Std. dev. & \pm 16.3 & \pm 14.1 & \pm 14.8 & \pm 16.6 & \pm 12.4 & \pm 11.0 & \pm 12.9 & - & - \\
\hline \hline
\end{tabular}

icant ( $p<0.001, p<0.001$, and $p<0.005$, respectively). The standard deviations among different classifiers in each of the feature sets decrease after the ReliefF step, which means that the selected subset of features is more robust and contains more useful information of the $\mathrm{SnS}$ compared to the original set. Finally, the best classification performance is achieved by a combination of all feature sets within a random forest classifier (UAR of 78\%). This reduced feature set has a dimension of 374, only $12.3 \%$ of the original feature set.

Fig. 4 provides an insight of the weight contribution of different types of features for the classification of $\mathrm{SnS}$. We found that, of all feature sets, WEF (containing WPTE and $W T E$ ), contributes most, followed by MFCCs and SFF. This is not surprising given the high dimension compared to the other feature sets. As for functionals, mean, max, and min values of the frame-based Low Level Descriptors contribute most, followed by the bias of the linear regression estimation. In addition, we illustrate the detailed weight contribution of $M F C C s$ and WTE. For WTE, we compare the contribution of the subsets by a level of decomposition. It is shown that the level-1 and level-2 decomposed components contribute most. In $M F C C s$, the $M F C C s-7$ and $M F C C s-2$ are best.

Fig. 5 shows the confusion matrix of the combination of all feature sets using a random forest classifier after ReliefF feature selection. We can see that among the four classes of SnS, Type $\mathrm{O}$ (the oropharyngeal area) and Type E (the epiglottis) are the two most easily wrongly classified as Type E and Type V (the level of the velum), respectively. In our experiments, the best trained classifier has the highest recognition accuracy for Type $\mathrm{T}$ (around 90\%) and the lowest accuracy for Type O (around $64 \%)$.

\section{Discussion}

In this paper, we systematically compare frequently used acoustic features for their performance on the classification of SnSs based on the VOTE model. In our experiments, we can achieve a UAR of $78 \%$ with the best combination of features and classifier. When performed by human experts, the interrater reliability of DISE classifications is up to $86 \%$ [53]. This is a benchmark for us, which has not yet been entirely achieved by our model. We believe that a major limitation is the small number of independent subjects in the database, and we aim to improve our results based on more data.

For our dataset of $\mathrm{SnSs}$ and with the experiments described, $M F C C s$ and WEF have shown to be the best suited feature sets (mean UAR at $68 \%$ and $66 \%$, respectively) across different classifiers. As a sophisticated indicator in speech recognition, $M F C C s$ can be regarded to represent the airway transfer function. In our previous studies on classification of different snore related sounds from overnight audio recordings, $M F C C s$ also proved to be quite efficient. Thus, MFCCs tend to play an important role $\mathrm{SnS}$ classification. 


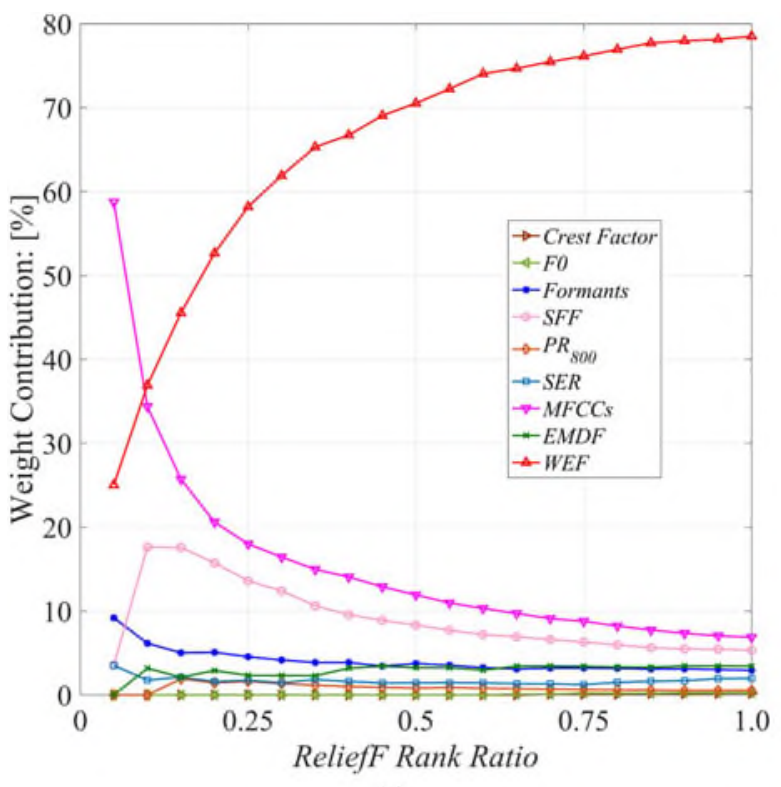

(a)

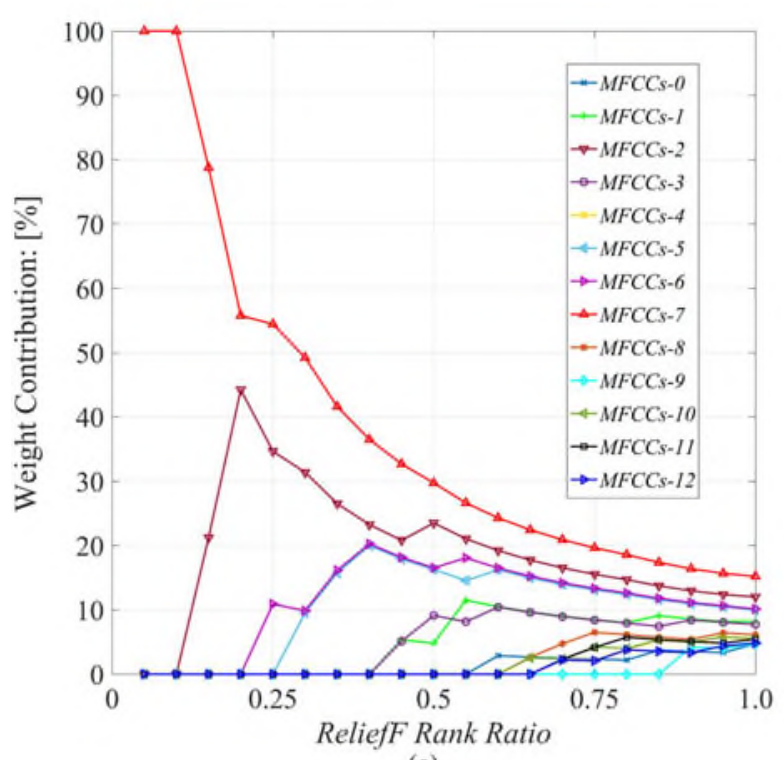

(c)

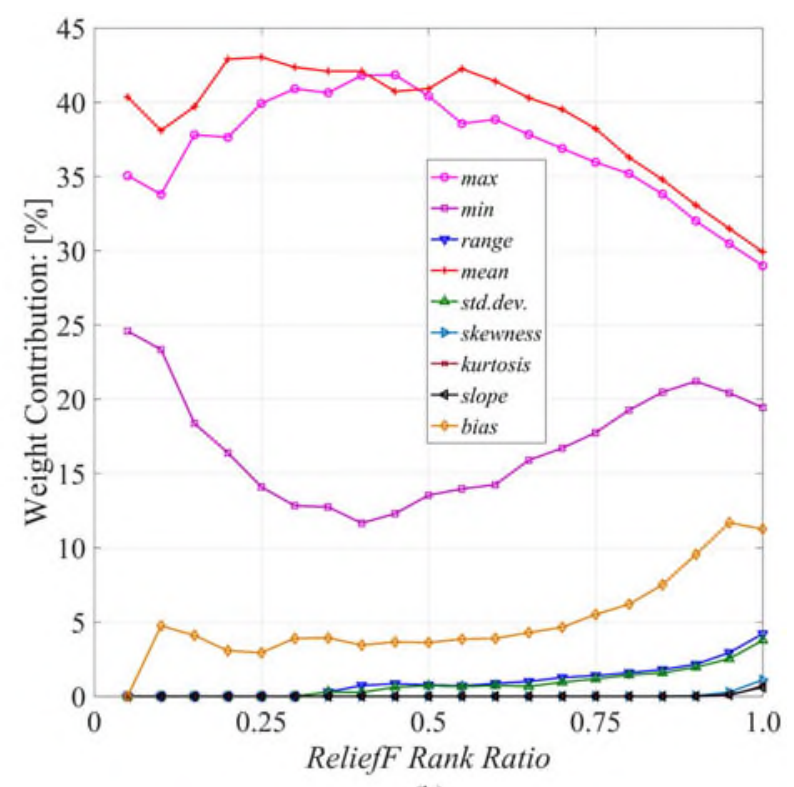

(b)

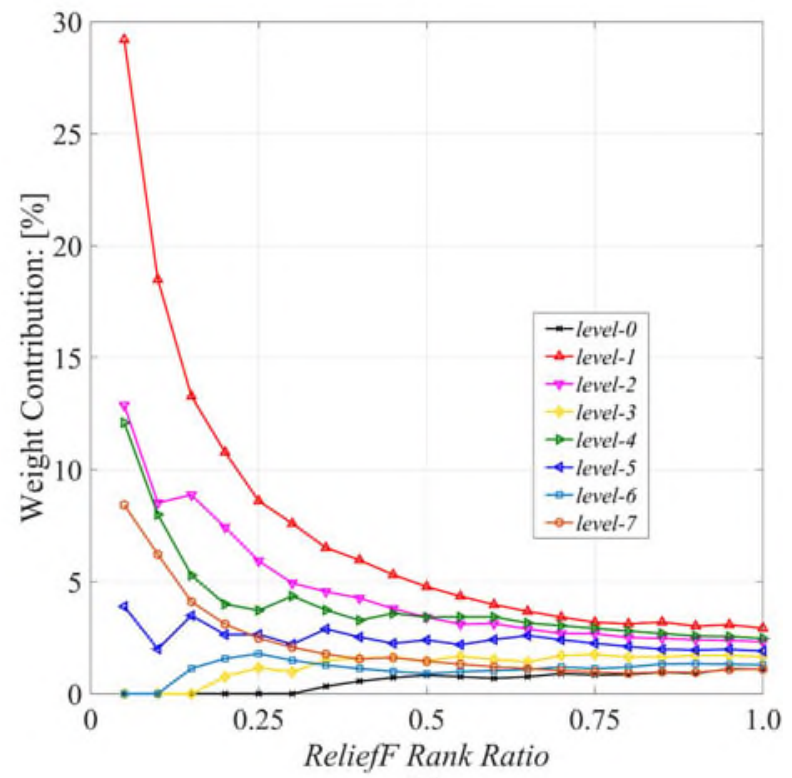

(d)

Fig. 4. Weight contribution ([\%]) analysis by the ReliefF algorithm with different Rank Ratios. (a) Detailed weight contribution of all feature sets. (b) Detailed weight contribution of functionals. (c) Detailed weight contribution of MFCCs. (d) Detailed weight contribution of WTE.

The proposed novel feature set $W E F$ is based on the wavelet transform theory, which is capable to give a multiresolution analysis of nonstationary signals. In the early works by Matsiki et al. [35], continuous wavelet transform is used to analyze the spectrum energy distribution changes before, during, and after apneic events based on snoring sounds. However, they did not propose a feature extraction method and their classification is not based on machine learning techniques. Furthermore, the number of subjects they investigated is small with only seven in total. $\mathrm{Ng}$ et al. [54] used wavelet polyspectral techniques to generate novel features to distinguish apneic from nonapneic snoring. Their results in [54] showed that wavelet-based features outperform the conventional spectral peak frequency. In our study, the
$W E F$ feature set, combining both the wavelet packet transform and the wavelet transform techniques, achieves an excellent performance of mean UAR of $66 \%$ among all classifiers used after the feature selection step. Further, it contributes most (69\%) in the best-performing feature set.

$\mathrm{Ng}$ et al. [55] describe that formants are representative of the physical frequency transfer function of the upper airway and can be good indicators to classify apneic and nonapneic snorers. The performance of the Formants feature set in our study is better than that of other features analyzed in earlier studies, i.e., Crest Factor, FO, and $\mathrm{PR}_{800}(p<0.001)$. This can be another indicator that the airway transfer function is a suitable indicator to distinguish different forms of snoring. 


\begin{tabular}{|c|c|c|c|c|c|}
\hline & & & asion 1 & & \\
\hline V & $\begin{array}{r}121 \\
24,2 \%\end{array}$ & 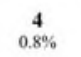 & $\begin{array}{c}1 \\
0.2 \%\end{array}$ & $\begin{array}{c}27 \\
5.4 \%\end{array}$ & $\begin{array}{l}79.1 \% \% \\
20.9 \%\end{array}$ \\
\hline $\mathrm{O}$ & $\begin{array}{c}10 \\
2.0 \%\end{array}$ & $\begin{array}{c}78 \\
15,6 \%\end{array}$ & $\begin{array}{c}1 \\
0.2 \%\end{array}$ & $\begin{array}{c}\mathbf{0} \\
0.0 \%\end{array}$ & $\begin{array}{l}87.6 \% \\
12.4 \%\end{array}$ \\
\hline & $\begin{array}{c}8 \\
1.6 \%\end{array}$ & $\begin{array}{c}\mathbf{0} \\
0.0 \%\end{array}$ & $\begin{array}{c}70 \\
14.0 \%\end{array}$ & $\begin{array}{c}\mathbf{3} \\
0.6 \%\end{array}$ & $\begin{array}{l}86.4 \% \\
13.6 \%\end{array}$ \\
\hline $\mathrm{E}$ & $\begin{array}{c}13 \\
2.6 \%\end{array}$ & $\begin{array}{c}40 \\
8.0 \%\end{array}$ & $\begin{array}{c}6 \\
1.2 \%\end{array}$ & $\begin{array}{c}118 \\
23.6 \%\end{array}$ & $\begin{array}{l}66.7 \% \% \\
33.3 \%\end{array}$ \\
\hline & $\begin{array}{l}79.6 \% \\
20.4 \%\end{array}$ & $\begin{array}{l}63.9 \% \\
36.1 \%\end{array}$ & $\begin{array}{c}89.7 \% \\
10.3 \%\end{array}$ & $\begin{array}{l}79.7 \% \\
20.3 \%\end{array}$ & $\begin{array}{c}77.4 \% \\
22.6 \%\end{array}$ \\
\hline & V & 0 & $\stackrel{\mathrm{T}}{\mathrm{T} \mathbf{c}}$ & E & \\
\hline
\end{tabular}

Fig. 5. Confusion matrix of the best-performing combination of feature set and classifier.

EMD is based on a different signal analysis method than the FFT. Specifically, it is suitable for nonstationary signals [32]. In this study, $E M D F$ did not prevail as a feature set. A possible reason for this moderate performance is that we based the classification task on relatively short fractions of a snoring event in order to increase the number of available segments for model learning. Therefore, the nonstationary characteristics of the underlying snore event could not be considered in full. A better performance can be expected when using this feature set on complete snoring events.

Future works can be done mainly in the following three areas: First, more potentially useful acoustic features can be tested, specifically, psychoacoustic characteristics (e.g., loudness, sharpness, roughness, and fluctuation strength), and higher order statistical model-based features (e.g., bispectrum), which have been studied in [56] and [57]. Some fundamental work to explore the relationship between feature properties and the anatomical changes in the upper airway can help to better understand the SnS generation mechanisms. Also, using complete snore events, rather than segments of snore sounds, as a basis for feature extraction can reveal additional useful information on the different snoring classes, as their nonstationary characteristics will show more clearly. A limitation of our work is the relatively small number of snoring subjects in the database used. Although, the total number of snore segments is sufficiently large to apply machine learning methods, they stem from comparably few different subjects. Last but not least, the feature selection phase at this stage of our method is based on an empirical parameter setting process (Rank Ratio is set using a step interval selection process) rather than on an automatic method without human involvement. Excluding the "human touch" on the parameter setting process will be important for future baseline improvement and practical product development.

\section{CONCLUSION}

For the first time, we comprehensively investigated various acoustic feature sets and classifiers for the task of classifying SnSs according to their excitation locations based on the VOTE model. Even with a relatively small dataset, we can achieve a good classification performance with selected feature sets independent of subjects. The results show that multifeature analysis is a promising means to help identifying the anatomical mechanisms of $\mathrm{SnS}$ generation in individual subjects.

\section{ACKNOWLEDGMENT}

The authors would like to thank all the colleagues involved in the collection of the labelled VOTE SnSs data.

\section{REFERENCES}

[1] P. E. Peppard et al., "Increased prevalence of sleep-disordered breathing in adults," Amer. J. Epidemiol., vol. 177, no. 9, pp. 1006-1014, 2013.

[2] P. J. Strollo Jr and R. M. Rogers, "Obstructive sleep apnea," New Engl. J. Med., vol. 334, no. 2, pp. 99-104, 1996.

[3] P. Smith et al., "Indications and standards for use of nasal continuous positive airway pressure (cpap) in sleep-apnea syndromes," Amer. J. Respiratory Critical Care Med., vol. 150, no. 6, pp. 1738-1745, 1994.

[4] T. Young et al., "The occurrence of sleep-disordered breathing among middle-aged adults," New Engl. J. Med., vol. 328, no. 17, pp. 1230-1235, 1993.

[5] B. Mokhlesi et al., "The effect of sex and age on the comorbidity burden of OSA: An observational analysis from a large nationwide US health claims database," Eur. Respiratory J., vol. 47, no. 4, pp. 1162-1169, 2016.

[6] M. S. Aldrich, Sleep Medicine. New York, USA: Oxford University Press, 1999.

[7] D. Pevernagie et al., "The acoustics of snoring," Sleep Med. Rev., vol. 14, no. 2, pp. 131-144, 2010.

[8] A. Roebuck et al., "A review of signals used in sleep analysis," Physiological Meas., vol. 35, no. 1, pp. R1-R57, 2014.

[9] K. K. Li, "Surgical therapy for adult obstructive sleep apnea," Sleep Med. Rev., vol. 9, no. 3, pp. 201-209, 2005.

[10] H.-C. Lin et al., "The efficacy of multilevel surgery of the upper airway in adults with obstructive sleep apnea/hypopnea syndrome," Laryngoscope, vol. 118, no. 5, pp. 902-908, 2008.

[11] M. R. El Badawey et al., "Predictive value of sleep nasendoscopy in the management of habitual snorers," Ann. Otol., Rhinol. Laryngol., vol. 112, no. 1, pp. 40-44, 2003.

[12] H. Demin et al., "Determining the site of airway obstruction in obstructive sleep apnea with airway pressure measurements during sleep," Laryngoscope, vol. 112, no. 11, pp. 2081-2085, 2002.

[13] M. Reda et al., "Pharyngoesophageal pressure monitoring in sleep apnea syndrome," Otolaryngology-Head Neck Surg., vol. 125, no. 4, pp. 324 331, 2001.

[14] B. A. Stuck and J. T. Maurer, "Airway evaluation in obstructive sleep apnea," Sleep Med. Rev., vol. 12, no. 6, pp. 411-436, 2008.

[15] S. Miyazaki et al., "Acoustic analysis of snoring and the site of airway obstruction in sleep related respiratory disorders," Acta Oto-Laryngologica, vol. 118, no. 537, pp. 47-51, 1998.

[16] P. Hill et al., "Palatal snoring identified by acoustic crest factor analysis," Physiological Meas., vol. 20, no. 2, pp. 167-174, 1999.

[17] S. Agrawal et al., "Sound frequency analysis and the site of snoring in natural and induced sleep," Clin. Otolaryngol. Allied Sci., vol. 27, no. 3, pp. 162-166, 2002.

[18] R. J. Beeton et al., "Snore site discrimination using statistical moment of free field snoring sounds recorded during sleep nasendoscopy," Physiological Meas., vol. 28, no. 10, pp. 1225-1236, 2007.

[19] C. Janott et al., "Akustische analyse von schnarchgeräuschen," Somnologie-Schlafforschung und Schlafmedizin, vol. 18, no. 2, pp. 8795, 2014.

[20] E. J. Kezirian et al., "Drug-induced sleep endoscopy: The vote classification," Eur. Arch. Oto-Rhino-Laryngol., vol. 268, no. 8, pp. 1233-1236, 2011.

[21] A. S. Karunajeewa et al., "Multi-feature snore sound analysis in obstructive sleep apnea-hypopnea syndrome," Physiological Meas., vol. 32, no. 1, pp. 83-97, 2011.

[22] K. Qian et al., "Comparison of two acoustic features for classification of different snore signals," Chin. J. Electron Devices, vol. 36, no. 4, pp. 455459, 2013.

[23] X. Sun, "Pitch determination and voice quality analysis using subharmonic-to-harmonic ratio," in Proc. IEEE Int. Conf. Acoust., Speech, Signal Process., Orlando, FL, USA, 2002, vol. 1, pp. 333-336. 
[24] J. R. Deller, Jr. et al., Discrete Time Processing of Speech Signals. Englewood Cliffs, NJ, USA: Prentice-Hall, 1993.

[25] T. Emoto et al., "Feature extraction for snore sound via neural network processing," in Proc. 29th Annu. Int. Conf. IEEE Eng. Med. Biol. Soc., Lyon, France, 2007, pp. 5477-5480.

[26] K. Qian et al., "Automatic detection of inspiration related snoring signals from original audio recording," in Proc. IEEE China Summit Int. Conf. Signal Inf. Process., Xi'an, China, 2014, pp. 95-99.

[27] W. Whitelaw, "Characteristics of the snoring noise in patients with and without occlusive sleep apnea," Amer. Rev. Respiratory Disease, vol. 147, pp. 635-644, 1993.

[28] M. Cavusoglu et al., "An efficient method for snore/nonsnore classification of sleep sounds," Physiological Meas., vol. 28, no. 8, pp. 841-853, 2007.

[29] A. Azarbarzin and Z. Moussavi, "Automatic and unsupervised snore sound extraction from respiratory sound signals," IEEE Trans. Biomed. Eng., vol. 58 , no. 5, pp. 1156-1162, May 2011

[30] L. R. Rabiner and R. W. Schafer, Theory and Application of Digital Speech Processing, Preliminary ed., Englewood Cliffs, NJ, USA: Prentice-Hall, 2009.

[31] K. Qian et al., "Automatic detection, segmentation and classification of snore related signals from overnight audio recording," IET Signal Process. vol. 9, no. 1, pp. 21-29, Feb. 2015.

[32] N. E. Huang et al., "The empirical mode decomposition and the Hilbert spectrum for nonlinear and non-stationary time series analysis," in Proc. Roy. Soc. London A, Math., Physical Eng. Sci., vol. 454, no. 1971, 1998, pp. 903-995.

[33] Y. Yu et al., "A roller bearing fault diagnosis method based on EMD energy entropy and ANN," J. Sound Vibration, vol. 294, no. 1, pp. 269-277, 2006.

[34] S. Mallat, A Wavelet Tour of Signal Processing. New York, NY, USA: Academic, 1999

[35] D. Matsiki et al., "Wavelet-based analysis of nocturnal snoring in apneic patients undergoing polysomnography," in Proc. 29th Annu. Int. Conf. IEEE Eng. Med. Biol. Soc., Lyon, France, 2007, pp. 1912-1915.

[36] A. K. Ng et al., "Snore signal enhancement and activity detection via translation-invariant wavelet transform," IEEE Trans. Biomed. Eng., vol. 55, no. 10, pp. 2332-2342, Oct. 2008.

[37] R. N. Khushaba et al., "Driver drowsiness classification using fuzzy wavelet-packet-based feature-extraction algorithm," IEEE Trans. Biomed. Eng., vol. 58, no. 1, pp. 121-131, Jan. 2011.

[38] R. R. Coifman et al., "Wavelet analysis and signal processing," in Wavelets and Their Applications. Sudbury, MA, USA: Jones and Barlett, 1992, pp. 153-178.

[39] K. B. Englehart, "Signal representation for classification of the transient myoelectric signal," Ph.D. dissertation, Dept. Elect., Comput., Eng., New Brunswick Univ., Fredericton, NB, Canada, 1998.

[40] K. Qian et al., "Wavelet features for classification of vote snore sounds," in Proc. IEEE Int. Conf. Acoust. Speech Signal Process., Shanghai, China, 2016, pp. 221-225.

[41] F. Eyben et al., "Opensmile: The Munich versatile and fast open-source audio feature extractor," in Proc. ACM Int. Conf. Multimedia., Firenze, Italy 2010, pp. 1459-1462.

[42] C. M. Bishop, Pattern Recognition and Machine Learning. New York, NY, USA: Springer-Verlag, 2006.

[43] I. Basheer and M. Hajmeer, "Artificial neural networks: Fundamentals, computing, design, and application," J. Microbiol. Methods, vol. 43, no. 1, pp. 3-31, 2000.

[44] V. Vapnik, The Nature of Statistical Learning Theory. Berlin, Germany: Springer Sci. Bus. Media, 2013.

[45] L. Breiman, "Random forests," Mach. Learn., vol. 45, no. 1, pp. 5-32, 2001.

[46] T. G. Dietterich, "Ensemble learning," The Handbook of Brain Theory and Neural Networks, vol. 2, Cambridge, MA, USA: MIT Press, 2002, pp. $110-125$.

[47] G.-B. Huang et al., "Extreme learning machine: Theory and applications," Neurocomput., vol. 70, no. 1, pp. 489-501, 2006.

[48] G.-B. Huang, "An insight into extreme learning machines: random neurons, random features and kernels," Cogn. Comput., vol. 6, no. 3, pp. 376390,2014

[49] C.-C. Chang and C.-J. Lin, "LIBSVM: A library for support vector machines," ACM Trans. Intell. Syst. Technol., vol. 2, pp. 27:1-27:27, 2011. [Online]. Available: http://www.csie.ntu.edu.tw/ cjlin/libsvm

[50] K. Kira and L. A. Rendell, "The feature selection problem: Traditional methods and a new algorithm," in Proc. 10th Nat. Conf. Artif. Intell., vol. 2, San Jose, CA, USA, 1992, pp. 129-134.

[51] I. Kononenko et al., "Overcoming the myopia of inductive learning algorithms with RELIEFF," Appl. Intell., vol. 7, no. 1, pp. 39-55, 1997.
[52] M. R. Spiegel et al., Probability and Statistics, New York, NY, USA: McGraw-Hill, 2009.

[53] E. J. Kezirian et al., "Interrater reliability of drug-induced sleep endoscopy," Archives Otolaryngol.-Head Neck Surg., vol. 136, no. 4 pp. 393-397, 2010

[54] A. K. Ng et al., "Investigation of obstructive sleep apnea using nonlinear mode interactions in nonstationary snore signals," Ann. Biomed. Eng., vol. 37, no. 9, pp. 1796-1806, 2009.

[55] A. K. Ng et al., "Could formant frequencies of snore signals be an alternative means for the diagnosis of obstructive sleep apnea?" Sleep Med., vol. 9, no. 8, pp. 894-898, 2008.

[56] M. Herzog et al., "Evaluation of acoustic characteristics of snoring sounds obtained during drug-induced sleep endoscopy," Sleep Breathing, 2014, pp. $1-9$.

[57] A. K. Ng et al., "Bispectral analysis of snore signals for obstructive sleep apnea detection," in Proc. 29th Annu. Int. Conf. IEEE Eng. Med. Biol. Soc., Lyon, France, 2007, pp. 6195-6198.

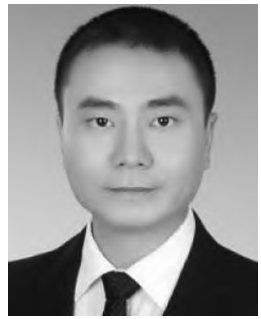

Kun Qian (S'14) received the Master's degree in signal and information processing from Nanjing University of Science and Technology, Nanjing, China, 2014. He is currently working toward the $\mathrm{Ph}$. D. degree in electrical engineering and information technology at the Technische Universität München (TUM), Munich, Germany. From November 2013 to April 2014, he was sponsored by a full fellowship of NUST for Master Program students (10 among 4600) to Nanyang Technological University, Singapore, as a Research Assistant in the Information Systems Research Laboratory. His research interests include signal processing, machine learning, biomedical engineering, and high performance computing system based machine learning

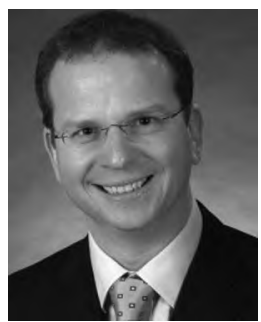

Christoph Janott (S'15) received the Dipl.Ing degree in electrical engineering from Technische Universität, Berlin, Germany, 1998.

$\mathrm{He}$ has held several leadership positions in product management and marketing in different technology companies. Since 2011, he has been coaching founders and young entrepreneurs in the field of medical devices in marketing and business development. $\mathrm{He}$ is the coauthor of several publications on speech intelligibility in classrooms and has co-invented several patents. He is currently a Doctoral Candidate in the Institute of Medical Engineering, Technische Universität München, Munich, Germany.

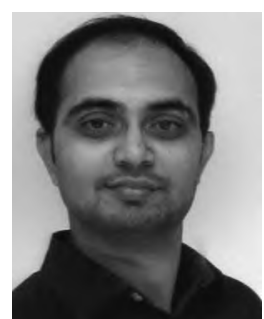

Vedhas Pandit (S'11) received the Master's degree from Arizona State University (ASU), Tempe, AZ, USA, in 2010 in electronic and mixed signal circuit design with his thesis on "Mathematical modeling of a-Si:H SOI transistors." Since February 2015, he has been working toward the Ph. D. degree at the University of Passau, Passau, Germany.

After working for Intel as a Graphics Hardware Engineer, he worked as a Researcher in the Indian Institute of Technology Bombay developing tools for automated music information retrieval, specifically for recurring pattern/melodic motif detection and identification, pattern classification, pattern variability characterization (e.g., nonuniform time warping, ornamentations) and investigating relevance of each to hindustani classical music tradition. His research interests include music information retrieval, speech and virtual instrument synthesis, deep learning strategies in machine learning, and biomedical signal processing. 


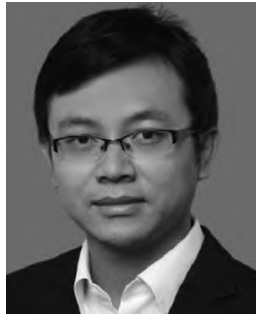

Zixing Zhang (M'15) received the Master's degree in physical electronics from Beijing University of Posts and Telecommunications, Beijing, China, in 2010, and the Ph. D. degree in engineering from the Institute for HumanMachine Communication, Technische Universität München, Munich, Germany, in 2015.

$\mathrm{He}$ is currently a Postdoctoral Researcher at the University of Passau, Passau, Germany. He has authored about 30 publications in peerreviewed journals and conference proceedings. His research interests include semisupervised learning, active learning, multitask learning, and deep learning in the application of computational paralinguistics, and automatic speech recognition.

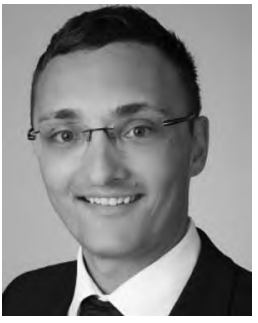

Clemens Heiser received the Graduate degree from the Medical School, University of Heidelberg, Heidelberg, Germany, in 2007. He began the residency in Otolaryngology (head and neck surgery) in Klinikum Mannheim, University of Heidelberg, Mannheim, Germany, and completed it in Klinikum Rechts der Isar (Technische Universität München), Munich, Germany, in 2011.

He completed his fellowship training in sleep medicine in Klinikum Mannheim, Mannheim, Germany. Since 2013, he has been the Head of the Sleep Department with Klinikum rechts der Isar, Munich, Germany. He is coauthor of the German guidelines in the treatment of snoring and Obstructive Sleep Apnea (OSA). His research interests include surgical treatment of snoring and OSA and developing surgical techniques and various alternative treatments for patients with OSA. Mr. Heiser is a Board Certified Member of the German Sleep Society.

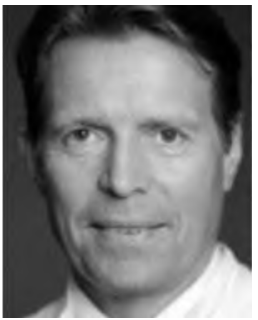

Winfried Hohenhorst received the Graduate degree from the Medical School, University of Essen, Duisburg, Germany, in 1985. He concluded his residency in anaesthesiology, otolaryngology and plastic surgery from Alfried Krupp Hospital, Essen, Germany, in 1992.

$\mathrm{He}$ is a Specialist for voice and speech disorders, allergology and plastic operations. Since 2012, he has been the Head of the clinic for ENT, plastic surgery, and operative sleep medicine with Alfried Krupp Hospital, Essen, Germany. He is coauthor of the German guidelines for the treatment of snoring and Obstructive Sleep Apnea (OSA). His research interests include diagnosis of sleep related breathing disorders by means of sleep endoscopy, as well as the surgical treatment of snoring and OSA. He is a Board Certified Member of the German Sleep Society.

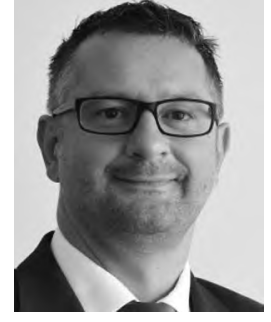

Michael Herzog received the Graduate degree from the Medical School, University of Würzburg, Würzburg, Germany, in 1998. He finished his residency in Otorhinolaryngology from the Department of ENT, University of Würzburg, Würzburg, Germany, in 2005.

From 2005 to 2015, he was a Senior Consultant in the Department of ENT, University of Greifswald, Germany, as well as Halle (Saale), Germany. Since 2015, he has been the Head of the Department of Otorhinolaryngology, Head and Neck Surgery, Carl-Thiem-Klinikum, Cottbus, Germany. He is a board certified member of the German Sleep Society and a Specialist for oncological, reconstructive, and plastic surgery. He is the coauthor of the German guidelines for the treatment of snoring and Obstructive Sleep Apnea. His research interests include diagnosis and surgical treatment of snoring, and sleep apnea, as well as the research in acoustic analysis of snoring sounds.

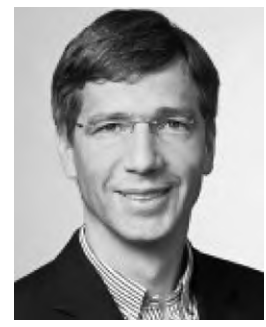

Werner Hemmert (M'00-SM'08) received the degrees in electrical engineering and computer engineering from Technische Universität München (TUM), Munich, Germany. He received the Doctorate degree from Ruhr-Universität Bochum, Bochum, Germany, in 1997.

After his studies, he continued his research career with the Tübingen Hearing Research Center (1991-1998), where he studied the cellular micromechanics of the inner ear. After that, he conducted research at the Massachusetts Institute of Technology (1998-2000), the IBM Research Laboratories in Zürich (2000-2001) and at Infineon Technologies, Corporate Research (2001-2007). He explored the principles of information processing in the auditory system and novel approaches for the advancement of neuroprostheses. His research interests include a combination of theoretical concepts and experiments, and he relies on close collaboration with workgroups from the fields of biology, medicine and industry. Since 2007, he has been a Professor for Bio-Inspired Information Processing with TUM. Prof. Hemmert is, among others, a Member of the German Acoustical Society, the Association for Research in Otolaryngology and the Graduate School of Systemic Neurosciences, and the Alexander von Humboldt Foundation.

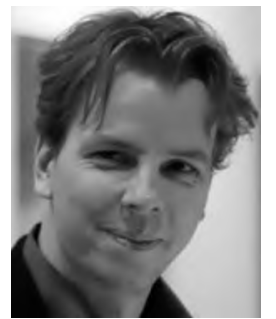

Björn Schuller (M'06-SM'15) received the Diploma degree in 1999, the Doctoral degree for his study on automatic speech and emotion recognition in 2006, and the Habilitation and Adjunct Teaching Professorship in the subject area of signal processing and machine intelligence in 2012, all in electrical engineering and information technology from Technische Universität München, Munich, Germany. He is currently a tenured Full Professor heading the Chair of Complex Systems Engineering, University of Passau, Passau, Germany, and a Reader (Associate Professor) in machine learning in the Department of Computing, Imperial College London, London, U.K. He has coauthored five books and more than 500 publications in peer-reviewed books, journals, and conference proceedings leading to more than 14000 citations.

Dr. Schuller is elected member of the IEEE Speech and Language Processing Technical Committee, Editor-in-Chief of the IEEE TRANSACTIONS ON A AFFECTIVE COMPUTING, and senior member of the ACM and ISCA. 